\title{
Características clínicas y comorbilidad psiquiátrica en jugadores patológicos de máquinas recreativas con premio, en tratamiento: Estudio de la población menor de 30 años
}

\author{
Ana Fernández-Alba Luengo.* \\ (*) Profesora Asociada. Universidad Complutense de Madrid. Facultad de Psicología. \\ Departamento de Personalidad, Evaluación y Tratamientos Psicológicos i (Psicología Clínica.) \\ Enviar correspondencia a:
}

Ana Fernández-Alba Luengo, Departamento de Personalidad, Evaluación y Tratamientos Psicológicos I (Psicología Clínica). Facultad de Psicología Universidad Complutense de Madrid. Campus de Somosaguas. 28223 Madrid. E-mail: anafalba@psi.ucm.es

\section{RESUMEN}

El objetivo de este estudio es la descripción de las características sociodemográficas, clínicas y la comorbilidad psiquiátrica que presenta una muestra, menor de 30 años de edad, de jugadores patológicos de máquinas recreativas con premio en tratamiento. La muestra está compuesta por 53 varones que cumplen los criterios DSM-IV de juego patológico. La edad media de la muestra se sitúa en los 22,89 años, siendo 17 la edad media con la que han comenzado a jugar a las máquinas recreativas con premio. Las principales formas de financiación del juego incluyen el préstamo de dinero realizado por personas del entorno del jugador $(67,9 \%)$, la adquisición de dinero familiar a través de cuentas bancarias $(56,6 \%)$, la comisión de robos a pequeña $(50,9 \%)$ y gran escala $(17 \%)$, la venta de droga o tabaco ilegal $(18,9 \%)$ o el uso de tarjetas de crédito/débito ajenas (11,3\%). El 47,1\% ha contraído algún tipo de deuda debido al juego. Ganar dinero es el motivo esgrimido con mayor frecuencia para comenzar a jugar $(45,3 \%)$ y recuperar el dinero perdido para continuar jugando (56,6\%). El $34 \%$ de la muestra refiere un consumo regular de sustancias ilegales, ya sea en el pasado o en el momento de acudir a tratamiento.

Palabras clave: juego patológico, máquinas recreativas con premio, psicopatología, consumo de sustancias psicoactivas, adolescentes, jóvenes

\section{ABSTRACT}

The purpose of this study is to describe the sociodemographic, clinical characteristics and psychiatric comorbility of a sample of pathological slot-machine gamblers, under 30 years of age, undergoing treatment. The sample comprises 53 men who met the DSM-IV criteria for pathological gambling. Mean age is 22.89 years, and the mean age at which they had started gambling on slot machines was 17 years. The main sources to finance their gambling include loans from people in the gambler's environment $(67.9 \%)$, taking money from family bank accounts $(56.6 \%)$, petty theft $(50.9 \%)$, serious theft $(17 \%)$, illegal tobacco and drug sales (18.9\%) or the use of someone else's credit card (11.3\%). Forty seven percent had incurred debts as a result of gambling. Winning money is the most frequent reason to begin gambling $(45.3 \%)$, and recovering lost money, the reason to continue to gamble $(56,6 \%)$. Thirtyfour percent of the sample regularly uses illegal substances, either in the past or when they commence treatment.

Key words: pathological gambling, slot machines, psychopathology, substance use, adolescence, young

\section{INTRODUCCIÓN}

D esde que en 1977 se produjera la legalización de los juegos de azar, el tapete nacional ha ido conformándose por una gran variedad de juegos de carácter privado y público, siendo estos últimos de índole estatal o autonómica. En la misma medida en

que ha crecido la oferta de juegos ha habido un incremento en las cantidades jugadas, hasta alcanzar cifras superiores a los veintiséis mil millones de euros en 
2001 (incremento interanual del 36,69\% desde 1996) (Comisión Nacional de Juego, 2002).

Estas cifras, en principio, reflejarían solamente la gran aceptación, difusión y práctica de los juegos

de azar en este país, no obstante, el carácter lúdico de esta actividad puede llegar a transformarse en un problema para una parte de la población de jugadores, lo que obliga a analizar el juego desde una perspectiva clínica. Los estudios epidemiológicos llevados a cabo en otros países, como Estados Unidos, Canadá, Reino Unido, Australia o Nueva Zelanda señalan una tasa de prevalencia del juego patológico que oscila, aproximadamente, entre el 1-2\% en la población adulta (Becoña y Míguez, 2001). A pesar de los pocos años transcurridos desde la legalización de los juegos de azar (en el caso de las máquinas recreativas con premio su legalización data de 1981), en España se han encontrado unas tasas de prevalencia similares (Arbinaga et al., 2000; Asociación de Jugadores de Azar en Rehabilitación del Campo de Gibraltar (JARCA), 1996; Becoña, 1993a,b; Becoña y Fuentes, 1995; Legarda, Babio y Andreu, 1992). Como indica Becoña (1996a), España ocupa, junto con Alemania, el primer puesto en gasto en juego per cápita de los países de la Comunidad Económica Europea y de los primeros del mundo, siendo previsible que la prevalencia de juego patológico se incremente en los próximos años.

Los estudios que analizan los hábitos de juego en el sector de la población menor de 18 años concluyen que el juego, sobre todo en ciertas modalidades, es una actividad cercana a los jóvenes; proximidad que ha ido adquiriendo en los últimos años un mayor arraigo, pues de unas cifras cercanas al $50 \%$ en la década de los 80 se ha pasado a otras que superan el $80 \%$ e incluso el $90 \%$ de niños y adolescentes que en alguna ocasión se han acercado a una máquina tragaperras, han participado en una partida de cartas, han comprado un número de lotería o han apostado en algún evento deportivo, sin descartar aquellos que se han deleitado con el glamour que irradian los salones de los casinos (Griffiths, 1995). Con un rango más amplio que en la población adulta, la tasa de prevalencia del juego patológico en niños y adolescentes oscila en las diferentes investigaciones entre el $1,5 \%$ y el $11,2 \%$ (Griffiths, 1995; Stinchfield et al., 1997). El meta-análisis realizado por Shaffer y Hall (1996), sobre los estudios desarrollados en Norteamérica, establece que entre el $4,4 \%$ y el $7,4 \%$ de los adolescentes manifiesta patrones de juego patológico, con otro 9,9-14,2\% que corre el riesgo de desarrollar un serio problema con el juego. Los estudios realizados en España arrojan unas tasas de prevalencia de juego patológico que rondan entre el 1,6\% en los escolares entre 11-16 años de la ciudad de Gijón (Villa, Becoña y Vázquez, 1997), el 2,23\% en la población entre 12-14 años de La Coruña (Becoña y Gestal, 1996), o el 2,7\% y el 2,9\% encon- trado en la población de Huelva cuya edad oscila entre 13-26 y 1417 años, respectivamente (Arbinaga, 1996a, b). Becoña y Míguez (2001) establecen que en la población de Galicia con edades comprendidas entre los 14-21 años la tasa de jugadores en riesgo alcanza el $8,2 \%$ y la de jugadores problema el $5,6 \%$ (estos autores recogen el cambio que ha sufrido la denominación del problema de juego en la población no clínica, sustituyendo el término de juego patológico por el de juego problema). A la mayor tasa de prevalencia de juego patológico en la población joven, hay que añadir un dato especialmente significativo extraído de los estudios realizados en nuestro país: el $40 \%$ de los jugadores patológicos adultos tiene una edad comprendida entre los 18-30 años (Becoña et al., 1995). Parece, por tanto, que la población joven constituye un grupo de riesgo a padecer problemas de juego. Por otro lado, de todos los juegos existentes en España, son las máquinas recreativas tipo B (esto es, las máquinas tragaperras ubicadas en bares y salas recreativas) las que contribuyen en mayor medida a las cifras astronómicas anteriormente citadas, constituyendo el $42 \%$ del importe de las cantidades jugadas en 2001; porcentaje que ha ido en aumento desde 1996, cuando ascendía al $34 \%$. Hay que destacar que el gasto realizado en las máquinas recreativas tipo $B$ supera la inversión correspondiente a la totalidad de los juegos públicos gestionados por el Organismo Nacional de Loterías y Apuestas del Estado (ONLAE) y la ONCE (Comisión Nacional del Juego, 2002). Las máquinas recreativas con premio se consolidan, por tanto, como unos de los juegos con más adeptos en países como España, donde su accesibilidad es manifiesta. Entre la población general de jugadores problema y patoló gicos la participación mayoritaria en este juego queda patente en dos datos destacables (Becoña, 1993b): (a) para el $50 \%$ de los jugadores patológicos las máquinas tragaperras constituyen su actividad de juego diario predominante y (b) el $43 \%$ dilapida su gasto de juego mensual máximo en las máquinas tragaperras. En la población de jugadores problema y patológicos que acuden a tratamiento también aparece una clara preferencia hacia este tipo de juego, porcentajes que oscilan entre un 75-84\% en España (Báez, Echeburúa y Fernández-Montalvo, 1994; Becoña et al., 1995). Entre las características que explican la atracción por las máquinas recreativas con premio caben destacar su accesibilidad -se encuentran tanto en locales de ocio como en bares y la cuantía de la apuesta no es elevada-, la inmediatez del refuerzo y el funcionamiento intrínseco de tales máquinas que propicia la ilusión de control. Su apariencia de un juego de habilidad (parecido estructural con los videojuegos) y su amplia difusión (incluidos locales comúnmente visitados por jóvenes y adolescentes) son algunos de los rasgos que favorecen que sea el juego elegido por antonomasia entre la población de menor edad. De hecho, los estudios realizados en Reino Unido ponen de manifiesto que las 
máquinas tragaperras constituyen no sólo el juego de apuestas más practicado sino una de las principales actividades de ocio entre la gente joven (Fisher, 1993; Griffiths, 1995).En diferentes estudios, tanto epidemiológicos como clínicos, se ha destacado la comorbilidad del juego patológico con otros trastornos, fundamentalmente el consumo excesivo de sustancias psicoactivas, entre las que sobresale el alcohol (Arbinaga, 2000; Becoña, 1993a; Lesieur, 1992; Lesieur, Blume y Zoppa, 1986; Llinares et al., 2002; McCormick et al., 1984; Ramírez et al., 1983; Rodríguez-Martos, 1989; Shaffer, Bilt y Hall, 1999; Smart y Ferris, 1996; Spunt et al., 1995, 1998; Steinberg, Kosten y Rounsaville, 1992a, b), y los trastornos depresivos (Blaszczynski y McConaghy, 1989; Lesieur, 1992; McCormick et al., 1984); así como la existencia de antecedentes familiares de problemas de juego y/o consumo excesivo de alcohol (American Psychiatric Association, APA, 1994; Ladouceur y Mireault, 1988; Lesieur, 1988). No obstante, la mayor parte de estos estudios se han realizado en la población adulta, siendo menor el número de investigaciones enfocadas a los adolescentes y jóvenes, a pesar de lo cual también se ha encontrado sistemáticamente en dicha población la relación entre problemas de juego y consumo excesivo de alcohol, tabaco o drogas ilegales (Arbinaga, 1996a, b; Becoña y Míguez, 2001; Ladouceur, Dubé y Bujold, 1994; Lesieur y Heineman, 1988).
Debido a que actualmente la población joven es la que sufre mayor riesgo de desarrollar un problema de juego, especialmente en máquinas recreativas con premio, y dado los escasos estudios realizados sobre esta población, el objetivo de la presente investigación es analizar, en la población de jugadores patológicos en tratamiento menores de 30 años, la manifestación clínica y la historia del problema de juego y su posible comorbilidad con otros síntomas y trastornos psicopatológicos.

\section{MÉTODO}

\section{Participantes}

La muestra está compuesta por 53 varones jugadores patológicos que demandaban asistencia por un problema de juego, cumpliendo los criterios DSM-IV para el diagnóstico de juego patológico y siendo su actividad de juego principal las máquinas recreativas con premio. La muestra original, perteneciente a un estudio realizado entre 1996-1999 (véase FernándezAlba, 1999; Fernández-Alba y Labrador, bajo revisión editorial), estaba compuesta por 88 sujetos (80 varones y 8 mujeres) cuyo rango de edad oscilaba entre los 1672 años ( $M=30,76$; DT $=12,23$ ), predominando los jugadores patológicos que no superan los treinta

Tabla 1. Estructura y contenido de la entrevista

\begin{tabular}{|c|c|}
\hline SECCIÓN & CONTENIDO \\
\hline Datos socio-demográficos & $\begin{array}{l}\text { Ficha de datos personales } \\
\text { - Nombre / Dirección / Teléfono } \\
\text {. Edad } \\
\text {. Estudios / Profesión / Trabajo } \\
\text {. Estado civil / No de hijos } \\
\text {. Clase social subjetiva } \\
\text {. Fuente que remite } \\
\text { - No de Historia Clínica }\end{array}$ \\
\hline $\begin{array}{l}\text { Diagnóstico de juego patológico y } \\
\text { trastornos del estado de ánimo }\end{array}$ & $\begin{array}{l}\text { Entrevista semiestructurada } \\
\text { - Diagnóstico DSM -IV de juego patológico } \\
\text { - Diagnóstico DSM -IV de trastornos del estado de ánimo } \\
\text { - Episodio maníaco/hipomaníaco } \\
\text { - Episodio depresivo mayor } \\
\text { - Trastorno distímico }\end{array}$ \\
\hline $\begin{array}{l}\text { Consumo pasado/presente } \\
\text { de sustancias adictivas }\end{array}$ & $\begin{array}{l}\text { Preguntas abiertas } \\
\text {. ¿Fuma? } \\
\text { ¿Toma algún tipo de droga? ¿Y en el pasado? } \\
\text { Frecuencia, duración e intensidad. } \\
\text { ¿ ¿Qué bebe mientras juega? } \\
\text { Cuestionario } \\
\text {. Cuestionario de alcoholismo CAGE. }\end{array}$ \\
\hline Datos referidos a la conducta de juego & $\begin{array}{l}\text { Entrevista conductual } \\
\text { - Análisis de secuencias actuales } \\
\text { - Historia clínica } \\
\text { - Importancia del problema } \\
\text { - Motivos por los que juega / Causalidad percibida } \\
\text { - Motivación hacia el cambio / Expectativas de cambio } \\
\text { - Recursos de autocontrol / Apoyo social }\end{array}$ \\
\hline
\end{tabular}


años de edad $(61,4 \%)$, que es la muestra objeto de análisis del presente estudio (se ha excluido de este estudio la única mujer que quedaba incluida en este rango de edad). Los pacientes fueron remitidos por distintas fuentes de la Comunidad de Madrid a la Facultad de Psicología de la Universidad Complutense donde se llevaba a cabo el programa de intervención (véase Fernández-Alba, 1999; Labrador y FernándezAlba, 2002), bien a través de servicios de asistencia social (e.g., teléfono de la esperanza, servicios del Ayuntamiento de Madrid, etc.), por profesionales de la salud o bien a través de asociaciones de autoayuda. Los criterios de exclusión incluían: (a) coexistencia de un problema de alcohol, (b) analfabetos o disminuidos psíquicos,

(c) motivaciones contrarias a la abstinencia al juego $u$ otras intenciones claras que no sean la de dejar de jugar, (d) seguir algún tipo de terapia para el tratamiento del juego patológico en el momento de estudio.

\section{Instrumentos de evaluación}

\section{Entrevista}

El contenido de la sesión de entrevista puede observarse en la Tabla 1.

La entrevista semiestructurada para el diagnóstico de juego patológico (véase Fernández-Alba y Labrador, 2002) está conformada por diez preguntas (algunas de las cuales se subdividen en otras adicionales si la respuesta es afirmativa) que evalúan cada uno de los criterios diagnósticos de inclusión propuestos por el DSM-IV (APA, 1994). En su elaboración se acudió a las siguientes fuentes: Ios criterios DSM-IV (APA, 1994), los criterios DSM-IVJ (Fisher, 1992), el Cuestionario de Juego de South Oaks (SOGS) (Lesieur y Blume, 1987) y la escala de detección de la adicción a las máquinas tragaperras (Becoña, 1996b).

Para el diagnóstico de los trastornos del estado de ánimo se utilizó la guía de entrevista realizada por Zimmerman (1994) y traducida a tal efecto por la autora de este estudio (véase Fernández-Alba y Labrador, 2001 b). Es una entrevista clínica no estandarizada que permite evaluar específicamente los trastornos objeto de estudio según los criterios DSM-IV en el contexto de una entrevista clínica. Esta entrevista semiestructurada consta de una serie de preguntas abiertas para evaluar los trastornos más comunes de los ejes I y II del DSM-IV (APA, 1994). En concreto, en el caso de los trastornos del estado de ánimo se comenzaba evaluando el diagnóstico de manía/hipomanía con objeto de excluir que las conductas de juego pudieran explicarse mejor por la presencia de un episodio maníaco; para continuar posteriormente con la evaluación de los criterios diagnósticos del trastorno depresivo mayor y del trastorno distímico.
En la detección de un posible problema de alcohol se empleó el Cuestionario de Alcoholismo CAGE (Hayfield, McLeod y Hall, 1974), en formato de entrevista. Es un instrumento de screening que consta de cuatro items con respuesta dicotómica. Con un punto de corte de 2, se obtiene una especificidad del 100\% y una sensibilidad del 96\% (Rodríguez-Martos, 1986).

Por último, se realizó una entrevista conductual sobre el problema de juego con objeto de evaluar los determinantes de la conducta de juego, la evolución del problema y las expectativas y objetivos del paciente. Para la confección de las preguntas sobre la conducta de juego se emplearon diversas fuentes como Becoña (1993b), Brown (1993), Echeburúa (1994) y Lesieur y Blume (1992).

\section{Cuestionario de Evaluación de Variables Depen- dientes del Juego (Echeburúa y Báez, 1991; citado en Echeburúa, 1994)}

A través de este inventario el paciente informa de la frecuencia, intensidad y duración de la conducta de juego en cada uno de los principales juegos de apuesta españoles y hace una valoración subjetiva de la frecuencia con la que juega, el dinero que gasta y el tiempo que invierte en el juego, así como de la frecuencia con la que piensa en el juego y su necesidad de jugar. Este cuestionario establece un criterio temporal para describir la conducta de juego que al ser semanal facilita la labor de concretar una cifra por parte del sujeto y, por tanto, favorece la fiabilidad y validez de dicha información.

\section{Inventario de Depresión de Beck, BDI (Beck, Rush, Shaw y Emery, 1979)}

Cuestionario autoaplicado que consta de 21 items y que evalúa síntomas depresivos. Cada item está compuesto por cuatro alternativas ordenadas de menor a mayor severidad (de 0 a 3 puntos), siendo la tarea del sujeto elegir aquella frase que se aproxime más a cómo se ha sentido durante la última semana. Los resultados en muestras españolas, en concreto en población con trastornos psicológicos, señalan que la fiabilidad es alta en términos de consistencia interna (coeficiente alfa de Cronbach $=0,90$ ) y, con un punto de corte de 21 puntos, la sensibilidad es del 75\% y la especificidad del 76\% (Vázquez y Sanz, 1999).

\section{Cuestionario de Ansiedad Estado-Rasgo, STAI (Spielberger, Gorsuch y Lushene, 1970)}

La situación de estrés que viven los jugadores patológicos y el hecho de que generalmente busquen ayuda terapéutica en momentos de crisis hacen necesario evaluar los niveles de ansiedad. Con este objetivo se aplicó la versión adaptada y traducida al castellano del STAI (subescala ansiedad-estado) (TEA, 1986), que consta de 20 items puntuables en una escala de cuatro puntos de 0 (nada) a 3 (mucho) a los 
que el sujeto debe responder informando sobre la intensidad con que aparecen en un momento concreto sentimientos o sensaciones de ansiedad. La fiabilidad calculada mediante el coeficiente alfa es de 0,90 (Miguel-Tobal, 1995).

\section{Procedimiento}

La aplicación de la entrevista, cuya duración aproximada era de hora y media, se realizaba exclusivamente con el sujeto sin la presencia de ningún familiar y enfatizando la confidencialidad de la información recibida.

Con objeto de no alargar excesivamente la entrevista con preguntas reiteradas y teniendo en cuenta la dificultad que experimentan los sujetos para concretar los parámetros de una conducta muy variable (al depender de condiciones externas no siempre controlables como la disposición de dinero o de tiempo) se decidió evaluar los parámetros de la conducta de juego con el Cuestionario de Evaluación de Variables Dependientes del Juego (versión para el paciente). Este cuestionario se aplicaba en la primera sesión del programa una vez concluida la entrevista. Al cumplimentarse en consulta, el terapeuta podía ayudar al paciente en su realización en caso de que fuera necesario.

Los cuestionarios BDI y STAl se entregaban al paciente al finalizar la sesión de entrevista clínica, con la instrucción de cumplimentarlos y traerlos en la siguiente sesión.

\section{RESULTADOS}

Variables sociodemográficas

\section{Edad}

La media de edad es de 22,89 años (DT $=4,02$ ), con un rango de edad que oscila entre los 16-30 años.

\section{Nivel de estudios}

El 11,3\% de la muestra no ha concluido los estudios primarios, un $32,1 \%$ ha completado el nivel de estudios primarios, el 49,1\% tiene estudios secundarios (Bachillerato o Formación Profesional), el 5,7\% de la muestra es diplomada y un 1,9\% licenciada universitaria.

\section{Situación laboral}

Las ocupaciones enmarcadas en el sector de servicios/ventas/seguridad, esto es, camareros, taxistas y transportistas, comerciales y vigilantes de seguridad $(34 \%)$; junto con estudiantes $(30,2 \%)$ y parados $(13,2 \%)$ son las tres situaciones laborales más representadas en la muestra. Las demás ocupaciones laborales de la muestra aparecen en un porcentaje menor: obrero sin cualificar (3,8\%), obrero cualificado (7,5\%), agricultor/ganadero (1,9\%), personal de servicios administrativos $(3,8 \%)$ y profesional/técnico $(1,9 \%)$.

\section{Estado civil}

El 88,7\% de la muestra está constituida por solteros, mientras el $11,3 \%$ restante lo conforman personas casadas o parejas de hecho.

Tabla 2. Criterios diagnósticos DSM-IV de juego patológico: porcentaje de la muestra que cumple cada síntoma.

\begin{tabular}{|c|c|c|c|}
\hline & $\begin{array}{l}\quad \text { Juego patológico } \\
\text { Criterios diagnósticos DSM -IV }\end{array}$ & $N$ & $\%$ \\
\hline 1 & $\begin{array}{l}\text { Preocupación por el juego (p. ej., preocupación por revivir experiencias pasadas de juego, compensar ventajas } \\
\text { entre competidores o planificar la próxima aventura, o pensar formas de conseguir dinero con el que jugar). }\end{array}$ & 44 & 83 \\
\hline 2 & Necesidad de jugar con cantidades crecientes de dinero para conseguir el grado de excitación deseado. & 45 & 84,9 \\
\hline 3 & Fracaso repetido de los esfuerzos para controlar, interrumpir o detener el juego. & 49 & 92,5 \\
\hline 4 & Inquietud o irritabilidad cuando intenta interrumpir o detener el juego. & 31 & 58,5 \\
\hline 5 & $\begin{array}{l}\text { El juego se utiliza como estrategia para escapar de los problemas o para aliviar la disforia (p. ej., sentimientos de } \\
\text { desesperanza, culpa, ansiedad, depresión). }\end{array}$ & 23 & 43,4 \\
\hline 6 & $\begin{array}{l}\text { Después de perder dinero en el juego, se vuelve otro día para intentar recuperarlo (tratando de "cazar» las propias } \\
\text { pérdidas). }\end{array}$ & 46 & 86,8 \\
\hline 7 & $\begin{array}{l}\text { Se engaña a los miembros de la familia, terapeutas u otras personas para ocultar el grado de implicación con } \\
\text { el juego }\end{array}$ & 53 & 100 \\
\hline 8 & Se cometen actos ilegales, como falsificación, fraude, robo, o abuso de confianza, para financiar el juego. & 51 & 96,2 \\
\hline 9 & $\begin{array}{l}\text { Se han arriesgado o perdido relaciones interpersonales significativas, trabajo y oportunidades educativas o } \\
\text { profesionales debido al juego. }\end{array}$ & 38 & 71,7 \\
\hline 10 & Se confía en que los demás proporcionen dinero que alivie la desesperada situación financiera causada por el juego. & 38 & 71,7 \\
\hline
\end{tabular}




\section{Número de hijos}

En la muestra predominan los sujetos que no tienen hijos (94,3\%); correspondiendo el $5,7 \%$ a los que tienen un hijo.

\section{Variables psicopatológicas}

\section{Diagnóstico de juego patológico}

En la Tabla 2 queda recogido el porcentaje de sujetos que cumplen cada uno de los criterios diagnósticos DSM-IV de juego patológico. Si bien todos los sujetos cumplen los criterios diagnósticos de juego patológico, entre el mínimo de cinco síntomas y el máximo de diez, la muestra se distribuye fundamentalmente entre los seis y nueve síntomas (84,9\%); en concreto, un 5,7\% de la muestra cumple cinco criterios, un $15,1 \%$ seis y siete síntomas respectivamente, el $22,6 \%$ ocho, el $32,1 \%$ nueve y el $9,4 \%$ diez criterios.

\section{Trastornos del estado de ánimo}

La mayor parte de la muestra $(67,9 \%)$ no presenta ningún trastorno del estado de ánimo. El 32,1\% restante se corresponde con alguna manifestación del trastorno depresivo mayor, principalmente la categoría de episodio único (leve: 18,9\%; moderado: 7,5\%) y en menor medida la categoría de episodio recidivante (leve: 1,9\%; moderado: 3,8\%).

\section{Sintomatología depresiva y ansiosa}

La puntuación media en el BDI es 16,02 (DT = 9,61), que atendiendo a los puntos de corte establecidos supone una depresión moderada. La puntuación media en el STAI-E es de 26,38 (DT = 13,34), que se corresponde con el percentil 65 en los baremos para adolescentes (población normativa adolescente: $\mathrm{M}=$ 22,35; DT = 11,03) y con el percentil 70 en los baremos para adultos (población normativa adulta: $\mathrm{M}=20,54$; $\mathrm{DT}=10,56)$.

\section{Consumo de sustancias psicoactivas}

Con respecto al consumo de sustancias, destaca el elevado porcentaje de la muestra que en el momento de acudir a consulta tiene el hábito de fumar $(88,7 \%)$. En lo referente al consumo de alcohol, el 3,8\% ha tenido problemas con la bebida en el pasado (recuérdese que el sufrir algún grado de alcoholismo en la actualidad era motivo de exclusión de la investigación). Por último, los sujetos refieren un consumo regular de sustancias ilegales, ya sea en el pasado o en el momento de acudir a tratamiento, que alcanza al 34\% de la muestra. El consumo regular de sustancias ilegales en el pasado se ciñe al uso de cannabis (18,9\%) y cocaína $(7,5 \%)$, y en menor medida a anfetaminas $(3,8 \%)$, alucinógenos $(1,9 \%)$ u opiáceos $(1,9 \%)$. El cannabis $(11,3 \%)$ y la cocaína $(1,9 \%)$ se configuran como las únicas drogas ilegales que los sujetos indican consumir en el momento de acudir a consulta.

Variables clínicas

\section{Parámetros de la conducta de juego}

La conducta de juego en las máquinas recreativas con premio se define por los siguientes valores medios: (a) frecuencia $\left(n^{\circ}\right.$ veces/semana): $M=8,25$ (DT = 7,84; rango = 1-50); (b) gasto mínimo de dinero (€/semana): $M=84,27$ (DT = 100,09; rango =6-450); (c) gasto máximo de dinero (€/semana): $M=155,13$ (DT = 154,57; rango $=12-601$ ); (d) tiempo invertido (horas/ semana): $M=11,82$ (DT = 9,7; rango $=0,4-42$ ).

En relación a otros juegos en los que la muestra ha participado con asiduidad se encuentran el bingo $(13,2 \%)$ y las cartas apostando $(15,1 \%)$, y en menor medida los juegos de casino $(3,8 \%)$, las loterías y la quiniela $(1,9 \%)$.

\section{Historia de juego}

La edad media de inicio en el juego rondaría los 15 años, elevándose a 17 la edad con la que se suele comenzar a jugar a las máquinas recreativas con premio, siendo de dos años aproximadamente la duración media de este problema hasta que acuden a tratamiento. Por su parte, cuando acuden a consulta, que por término medio es el primer tratamiento que reciben, suelen llevar unos nueve días sin jugar, han conseguido, en alguna ocasión, permanecer abstinentes aproximadamente dos meses, $y$, por término medio, han experimentando una recaída en su relación con las máquinas recreativas con premio (véase Tabla 3).

Tabla 3. Media, desviación típica y rango de las principales variables de la historia de juego

\begin{tabular}{|c|c|c|c|c|}
\hline & \multirow[b]{2}{*}{ M } & \multirow[b]{2}{*}{ DT } & \multicolumn{2}{|c|}{ Rango } \\
\hline & & & Mín & Máx \\
\hline Edad a la que comenzó a jugar (años) & 15,17 & 4,79 & 5 & 30 \\
\hline $\begin{array}{l}\text { Edad a la que comenzó a jugar a las } \\
\text { máquinas recreativas con premio } \\
\text { (años) }\end{array}$ & 17,4 & 3,27 & 9 & 30 \\
\hline $\begin{array}{l}\text { Duración del problema (máquinas } \\
\text { recreativas con premio) (meses) }\end{array}$ & 23,13 & 25,19 & 3 & 120 \\
\hline $\begin{array}{l}\text { Tiempo que lleva sin jugar a las máqui- } \\
\text { nas cuando acude a consulta (días) }\end{array}$ & 9,41 & 10,74 & 0 & 60 \\
\hline $\begin{array}{l}\text { Número de recaídas (máquinas recrea - } \\
\text { tivas con premio) }\end{array}$ & 0,98 & 1,56 & 0 & 9 \\
\hline $\begin{array}{l}\text { Duración del último período voluntario } \\
\text { de abstinencia (máquinas) (días) }\end{array}$ & 66,27 & 94,68 & 0 & 365 \\
\hline Número de tratamientos anteriores & 0,34 & 0,62 & 0 & 3 \\
\hline
\end{tabular}


Con respecto a la forma de financiarse el juego, hay que indicar que el 96,2\% de la muestra emplea el dinero de su paga o su sueldo para jugar. En cuanto a otras formas de conseguir dinero con el que jugar, el préstamo de dinero realizado por personas conocedoras (encargados en establecimientos de hostelería) o no (familiares, amigos...) del destino de ese dinero se configura en la fuente principal de suministro para el jugador $(67,9 \%)$, seguido por la adquisición de dinero familiar (por ejemplo, de cuentas conjuntas) sin el conocimiento del implicado o ignorando éste su final $(56,6 \%)$ y por la comisión de robos a pequeña escala (por ejemplo, coger dinero de casa o quedarse con un dinero que tenía otro fin) que los lleva a cabo el 50,9\% de la muestra. Otros medios para financiarse el juego alternativos a los anteriores son la venta de droga o tabaco ilegal $(18,9 \%)$, cometer robos de dinero a gran escala, esto es, en tiendas o en el trabajo, para financiarse el juego (17\%) o el uso de tarjetas de crédito/ débito ajenas $(11,3 \%)$. El resto de las actividades son realizadas por un porcentaje inferior al 10\% de la muestra: cometer alguna falsificación $(7,5 \%)$ o fraude $(3,8 \%)$, solicitar un crédito o acudir a prestamistas en un $5,7 \%$, vender objetos propios $(3,8 \%)$ o ajenos $(1,9 \%)$, vender acciones $u$ otros valores bancarios $(1,9 \%)$ o propiedades personales o familiares $(1,9 \%)$ o solicitar anticipos en el trabajo (1,9\%). Por término medio los sujetos emplean tres de estos medios para financiarse el juego $(M=3,47 ;$ DT $=1,31$; rango $=1$ 7).

Otro dato económico relevante son las deudas. En esta muestra el $47,1 \%$ ha contraído algún tipo de deuda, aunque de ese porcentaje el 7,5\% han sido saldadas por alguien del entorno del jugador.

En la Tabla 4 puede observarse la discrepancia entre los motivos esgrimidos por los jugadores para comenzar a jugar y las razones por las que continúan jugando. Otro dato relevante es que el 67,9\% recuerda que en las primeras ocasiones en las que jugaba a las máquinas recreativas con premio obtuvo uno de los premios de mayor cuantía.

Tabla 4. Motivos esgrimidos para jugar a las máquinas recreativas con premio (en porcentaje)

\begin{tabular}{lrrrr}
\hline & $\begin{array}{c}\text { Motivos por los } \\
\text { que se comenzó } \\
\text { a jugar }\end{array}$ & \multicolumn{2}{c}{$\begin{array}{c}\text { Motivos por los } \\
\text { que continúa } \\
\text { jugando }\end{array}$} \\
\hline Ganar dinero & $\mathrm{N}$ & $\%$ & $\mathrm{~N}$ & $\%$ \\
Entretenimiento & 24 & 45,3 & 24 & 45,3 \\
Personas que le incitaban & 13 & 24,5 & 15 & 28,3 \\
Evasión de problemas & 3 & 5,7 & 0 & 0 \\
Algo prohibido & 1 & 1,9 & 12 & 22,6 \\
Excitación & 2 & 3,8 & 0 & 0 \\
Ser sociable & 2 & 3,8 & 4 & 7,5 \\
Recuperar un dinero perdido & 0 & 13,2 & 0 & 0 \\
Otros & 7 & 0 & 30 & 56,6 \\
\hline
\end{tabular}

Al rastrear entre los jugadores de la muestra la presencia de antecedentes familiares con problemas de juego, alcohol y/o drogas, se encuentran antecedentes familiares con problemas de juego $(32,1 \%)$ y alcohol $(32,1 \%)$ en mayor medida que de adicción a sustancias ilegales (11,3\%). En el caso de los antecedentes de juego fundamentalmente es el padre el que tiene dicho problema $(11,3 \%)$ y en menor medida los hermanos $(3,8 \%)$, la madre $(1,9 \%)$, los abuelos $(1,9 \%)$, otros familiares $(5,7 \%)$ o varios antecedentes (7,5\%). En el caso de problemas de alcohol, de nuevo destaca la figura paterna $(11,3 \%)$, la madre o los hermanos alcanzan un 1,9\%, respectivamente, los abuelos un $5,7 \%$ y otros familiares el $11,3 \%$. El 11,3\% de la muestra que afirma tener antecedentes familiares con problemas de adicción a las drogas ilegales se corresponde con otro tipo de familiares que no son ni los progenitores, ni los hermanos ni los abuelos.

\section{DISCUSIÓN}

Estudios previos realizados con jugadores patoló gicos en tratamiento (Báez et al., 1994; Ciarrocchi y Richardson, 1989; Jiménez y Fernández, 1999; Legarda et al., 1992; Volberg, 1994; Volberg y Steadman, 1988) destacan cómo en esta población clínica hay un predominio del rango de edad comprendido entre los 30-50 años. No obstante, en la presente investigación los jugadores patológicos que no superan los 30 años de edad suponen el $61,4 \%$ de la muestra completa original (véase Fernández-Alba, 1999; Fer-nández-Alba y Labrador, bajo revisión editorial). Este predominio de jugadores con menos de treinta años podría ser resultado de un sesgo en el proceso de captación de los sujetos, ya que una de las principales fuentes de remisión de sujetos (un grupo de auto-ayuda) tendía a remitir a este programa de tratamiento a los jugadores con problemas más jóvenes, debido a que esta población tenía dificultades para sentirse identificada en un grupo cuyos miembros, por su edad más avanzada, compartían una serie de problemas como consecuencia del juego que poco o nada tenían que ver con los suyos. En cualquier caso, aún cuando la población menor de treinta años pueda estar sobrerrepresentada en este estudio, es un dato que coincide con los ofrecidos por las investigaciones realizadas en la población general.

Relacionado con la juventud de la muestra, hay que destacar que la media de inicio en los juegos de azar ronda los quince años y los diecisiete en el caso de las máquinas recreativas con premio, cuando su acceso no está permitido a los menores de 18 años, repitiendo un patrón similar al del consumo de tabaco y alcohol. Por otro lado, además de las máquinas tragaperras, las cartas apostando y el bingo son los juegos de azar en 
los que más participa esta muestra, una elección en la que pueden estar influyendo tanto la facilidad de acceso al juego como la inmediatez del refuerzo.

Por el número y el tipo de síntomas de juego patológico que padecen los sujetos, junto con el hecho de que el $47,1 \%$ de la muestra ha contraído alguna deuda, puede afirmarse que la mayor parte de estos pacientes, a pesar de su edad, acuden al psicólogo cuando el trastorno ya se ha desarrollado y manifiesta cierta gravedad, siendo la duración media de este problema, estimada por los propios pacientes, de dos años aproximadamente. Aunque todos los criterios DSMIV de juego patológico se cumplen en un porcentaje que supera el $70 \%$ de la muestra, a excepción de los referidos a la experiencia de inquietud o irritabilidad cuando se intenta detener el juego (criterio 4: 58,5\%) y el uso del juego como forma de escape o alivio de la disforia (criterio 5: 43,4\%), los más prevalentes son aquellos que tienen que ver con ocultar información o engañar sobre la propia implicación en el juego (criterio 7: 100\%), la comisión de actos ilegales u otro tipo de acciones para financiarse el juego (criterio 8: $96,2 \%$ ), así como la caza de la propias pérdidas (criterio 6: $86,8 \%)$.

Analizado la forma de financiarse el juego, por término medio los sujetos tienen tres fuentes de obtención de dinero, de las cuales la prioritaria suele ser su sueldo o paga, no obstante, pueden llegar a realizar determinadas acciones, fundamentalmente robo de dinero en el ámbito familiar, robos a pequeña escala o, incluso, en tiendas o en el trabajo, así como el uso de tarjetas de crédito/débito ajenas y la venta de droga o tabaco ilegal. Estos datos ratifican el alcance de una actividad que se acompaña en un número notable de ocasiones de conductas delictivas concomitantes que, en el mejor de los casos, no siendo un delito constituyen una infracción digna de mención, fundamentalmente debido a la regularidad con la que se llevan a cabo (Fisher, 1992; Griffiths, 1995). Conductas que, además, contribuyen a alterar la vida escolar o laboral de esta población; cifras que según Ladouceur y Mireault (1988) Ilegan al 6,2\% de los entrevistados que admiten tener problemas en estas áreas debido al juego.

Relacionado con la venta de droga o tabaco ilegal para financiarse el juego, hay que indicar que además de vender, el $34 \%$ de la muestra refiere un consumo regular de sustancias ilegales, ya sea en el pasado o en el momento de acudir a tratamiento, además de que el $88,7 \%$ consume tabaco y el $3,8 \%$ ha tenido problemas con el alcohol en el pasado. La comorbilidad del juego y el consumo de sustancias psicoactivas en la población adolescente ya había sido constatada en nuestro país, entre otros autores, por Arbinaga (1996a) y Becoña y Míguez (2001). Los resultados también señalan la existencia de cierta sintomatología depresiva y, en menor medida, ansiosa concomitante al juego patológico en los adolescentes y jóvenes.

Resulta interesante detenerse en la situación laboral de la muestra, concretamente en dos aspectos. El primero de ellos haría referencia al porcentaje de sujetos de la muestra cuya actividad laboral puede catalogarse en el amplio sector de servicios (camareros, taxistas, transportistas, comerciales), ocupaciones que se corresponden con la situación laboral más repetida en esta muestra (34\%). Es posible que la preponderancia de este gremio se deba a que incluye mayor variedad de profesiones que el resto de las categorías establecidas, pero también cabe presuponer que la profesión de camarero se desarrolla en un entorno de trabajo donde las máquinas recreativas están presentes, favoreciendo un primer acercamiento a las mismas. De igual manera, si hubiera que encontrar un factor común en taxistas, transportistas o, incluso, comerciales sería la gran cantidad de tiempo que estos profesionales pasan en la calle, lo que probablemente también suponga frecuentar en mayor medida cafeterías, bares..., y las máquinas recreativas que se encuentran en estos locales. El otro aspecto destacable es que la segunda y tercera posición en este ranking de actividades laborales desempeñadas por la muestra son ocupadas por estudiantes $(30,2 \%)$ y desempleados $(13,2 \%)$, respectivamente. En realidad, no sorprende el alto porcentaje de estudiantes y parados per se, sino la ausencia de ingresos que ello supone, planteando el interrogante de cómo se financian el juego. ¿Cuáles son sus fuentes de obtención de dinero?. "No parece excesivamente alarmista pensar que, probablemente, esta población se vea avocada con mayor celeridad a usar ciertos procedimientos para financiarse el juego que, constituyan o no un delito, son infracciones destacables y que, en definitiva, sólo contribuyen a acelerar la espiral de deterioro del jugador" (Fernández-Alba y Labrador, 2001a, p. 21). Si a ello se le añade la escasa edad de esta población, se puede especular que, además de comenzar a jugar más tempranamente, en los jóvenes esta actividad se convierte con mayor celeridad en un problema.

Algunos de los modelos explicativos de la conducta de juego (Jacobs, 1986; McCormick y Ramírez, 1988) consideran esta actividad como resultado de un déficit o carencia; no obstante, los propios jugadores, al menos entre los varones, dan explicaciones más sencillas a su conducta de juego: juegan, fundamentalmente, por ganar dinero (o recuperarlo), y como forma de entretenimiento. La realización de esta actividad con el fin de rehuir u olvidarse de los problemas resulta un motivo destacable cuando el jugador ya ha entrando en la espiral de deterioro, siendo muy probable que esos problemas que intenta olvidar con el juego sean resultado de esta misma actividad. De hecho, el criterio diagnóstico que hace referencia a la 
utilización del juego como estrategia para escapar de los problemas o aliviar la disforia, es el que no se cumple en un porcentaje mayor de la muestra. En definitiva, estos datos resaltan la capacidad de atracción del juego en sí mismo, al menos para los varones; y, como consecuencia de ello, el acercamiento a esta actividad suele deberse a los aspectos positivos que ofrece la misma: ganar dinero y entretenimiento. En este punto, además, es destacable el $67,9 \%$ de la muestra que recuerda haber ganado uno de los premios de mayor cuantía en las primeras ocasiones en las que se jugó a las máquinas recreativas. En el estudio de Arbinaga (1996b) se obtienen unos resultados similares en una muestra de estudiantes menores de 18 años: "el $21,90 \%$ de los sujetos reconocen jugar para conseguir dinero, en el caso de jugar por entretenimiento lo hacen el $76,19 \%$, el $36,19 \%$ porque lo hacen sus amigos, el 18,09\% para obtener sensaciones, el 21,90\% para olvidarse de los problemas y el $2,9 \%$ porque no puede dejar de jugar" (p. 342).

Debido a que las tasas de prevalencia de juego patológico y problema en la población que no supera los 30 años de edad son más elevadas que las referidas a otros estratos de edad, parece necesario realizar estudios centrados en este grupo de riesgo. En este sentido, ulteriores investigaciones deberían prestar especial atención a la prevención y el tratamiento de esta población clínica con características peculiares debido a la edad. Por otro lado, parece pertinente realizar estudios sobre la mujer joven con problemas de juego, ya que los cambios sociales experimentados en las últimas décadas podrían estar contribuyendo a un mayor acercamiento de la mujer al juego y, además, en unas condiciones similares a las del varón, reduciéndose ciertas diferencias clínicas encontradas entre ambos sexos en la población de mediana edad (Becoña, 1997; Mark y Lesieur, 1992).

\section{REFERENCIAS}

American Psychiatric Association (1994/1995). DSM-IV. Manual diagnóstico y estadístico de los trastornos mentales. Barcelona: Masson.

Arbinaga, F. (1996a). Conductas de juego con apuestas y uso de drogas en una muestra de estudiantes adolescentes de la ciudad de Huelva. Análisis y Modificación de Conducta, 22, 577-601.

Arbinaga, F. (1996b). El juego patológico en estudiantes menores de 18 años: incidencia, uso de drogas y variables asociadas. Adicciones, 8, 331-347.

Arbinaga, F. (2000). Consumo de drogas y juego patológico en Punta Umbría (Huelva). Revista Española de Drogodependencias, 25, 301-320.
Arbinaga, F., Arbinaga, A.M., Albende, M.J., López, P.L. Y Sánchez, C. (2000). Juegos de apuesta y juego patológico en Punta Umbría (Huelva): Prevalencia y características. Análisis y Modificación de Conducta, 26, 257-287.

Báez, C., Echeburúa, E. y Fernández-Montalvo, J. (1994). Características demográficas de personalidad y psicopatología de los jugadores patológicos de máquinas tragaperras en tratamiento: un estudio descriptivo. Clínica y Salud, 5, 289-305

Beck, A.T., Rush, A.J., Shaw, B.F. y Emery, G. (1983). Terapia cognitiva de la depresión. Bilbao: Desclée de Brower (original 1979).

Becoña, E. (1993a). The prevalence of pathological gambling in Galicia (Spain). Journal of Gambling Studies, 9, 353-369.

Becoña, E. (1993b). El juego compulsivo en la Comunidad Autónoma Gallega. I. Ciudades. Xunta de Galicia: Servicio Galego de Saúde.

Becoña, E. (1996a). Prevalence surveys of problem and pathological gambling in Europe: The cases of German, Holland and Spain. Journal of Gambling Studies, 12, 179-192.

Becoña, E. (1996b). La ludopatía. Madrid: Aguilar.

Becoña, E. (1997). Características de la mujer jugadora patológica. Revista de Psicopatología y Psicología Clínica, 2, 21-34.

Becoña, E. y Fuentes, M.J. (1995). El juego patológico en Galicia evaluado por el South Oaks Gambling Screen. Adicciones, 7, 423-440.

Becoña, E., Labrador, F., Echeburúa, E., Ochoa, E. y Vallejo, M.A. (1995). Slot machine gambling in Spain: An important and new social problem. Journal of Gambling Studies, 11, 265-286.

Becoña, E. y Gestal, C. (1996). El juego patológico en niños del $2^{\circ}$ ciclo de E.G.B. Psicothema, 8, 13-23.

Becoña, E. y Míguez, M.C. (2001). Juego problema y juego de riesgo en adolescentes: su relación con el consumo de tabaco y alcohol. Adicciones, 13, 279-288.

Blaszczynski, A. y McConaghy, N. (1989). Anxiety and/or depression in the pathogenesis of addictive gambling. The International Journal of the Addictions, 24, 337350.

Brown, R.I.F. (1993). El papel de la activación, distorsiones cognitivas y búsqueda de sensaciones en las adicciones al juego. Psicología Conductual, 1, 375-388.

Ciarrocchi, J. y Richardson, R. (1989). Profile of compulsive gamblers in treatment: Update and comparisons. Journal of Gambling Behavior, 5, 53-65.

Comisión Nacional del Juego (2002). Memoria 2001. Ministerio del Interior: Comisión Nacional del Juego.

Echeburúa, E. (1994). Evaluación y tratamiento de los trastornos adictivos. Madrid: Fundación Uni-versidadEmpresa.

Fernández-Alba, A. (1999). Tratamiento psicológico del juego patológico en jugadores de máquinas recreativas con premio. Tesis Doctoral: Universidad Complutense de Madrid. 
Fernández-Alba, A. y Labrador, F.J. (2001a). Comisión de actos delictivos en una muestra de jugadores patológicos de máquinas recreativas con premio: datos empíricos. Psicología Clínica, Legal y Forense, 1, 5-25.

Fernández-Alba, A. y Labrador, F.J. (2001b). Juego patológico en la Comunidad de Madrid. Madrid: Consejo Económico y Social. Comunidad de Madrid.

Fernández-Alba, A. y Labrador, F.J. (2002). Juego patológico. Madrid: Síntesis.

Fernández-Alba, A. y Labrador, F.J. (en prensa). Sociodemographic, Psychopathological and Clinical Characteristics of a Sample of Pathological Slot-Machine Gamblers in Treatment: A Descriptive Study. Journal of Gambling Studies.

Fisher, S. (1992). Measuring pathological gambling in children: The case of fruit machines in the U.K. Journal of Gambling Studies, 8, 263-285.

Fisher, S. (1993). Gambling and pathological gambling in adolescents. Journal of Gambling Studies, 9, 277-288.

Griffiths, M.D. (1995). Adolescent gambling. London: Routledge.

Hayfield, D., McLeod, G. y Hall, P. (1974). The CAGE questionnaire validation of a new alcoholism screening instrument. American Journal of Psychiatry, 131, 1121-1123.

Jacobs, D.F. (1986). A general theory of addictions: A new theoretical model. Journal of Gambling Behavior, 2, 15-31.

JARCA (1996). Estudio sobre el juego de azar en Algeciras: prevalencia y características socio-demográficas. Asociación de Jugadores de Azar en Rehabilitación del Campo de Gibraltar.

Jiménez, J. y Fernández, E. (1999). Estudio de algunas variables sociodemográficas relevantes en jugadores patológicos que acuden a tratamiento. Análisis y Modificación de Conducta, 25, 51-80.

Labrador, F.J. y Fernández-Alba, A. (2002). Treatment of pathological gambling. En C. von Hofsten y

L. Bäckman (Eds.), Psychology at the turn of the millennium (vol. 2, pp. 271-301). East Sussex and New York: Psychology Press.

Ladouceur, R. y Mireault, A. (1988). Gambling behaviors among high school students in the Quebec area. Journal of Gambling Behavior, 4, 3-12.

Ladouceur, R., Dubé, D. y Bujold, A. (1994). Prevalence of pathological gambling and related problems among college students in the Quebec Metropolitan Area. Canadian Journal of Psychiatry, 39, 289-293.

Legarda, J.J., Babio, R. y Abreu, J.M. (1992). Prevalence estimates of pathological gambling in Seville (Spain). British Journal of Addiction, 87, 767-770.

Lesieur, H.R. (1988). Report and recommendations of the Governor's Advisory Commission on Gambling. Trenton, New Jersey.

Lesieur, H.R. (1992). Compulsive gambling. Social Science and Modern Society, 29, 43-50.

Lesieur, H.R., Blume, S.B. y Zoppa, R.M. (1986). Alcoholism, drug abuse, and gambling. Alcoholism: Clinical and Experimental Research, 10, 33-38.
Lesieur, H.R.y Blume, S.B. (1987). The South Oaks Gambling Screen (SOGS): A new instrument for the identification of pathological gamblers. American Journal of Psychiatry, 144, 1184-1188.

Lesieur, H.R. y Heineman, M. (1988). Pathological gambling among youthful multiple substance abusers in a therapeutic community. British Journal of Addictions, 83, 765-771.

Lesieur, H.R. y Blume, S.B. (1992). Modifying the Addiction Severity Index for use with pathological gamblers. American Journal on Addictions, 1, 240247.

Llinares, M.C., Palau, C., Santos, P., Albiach, C. y Camacho, I. (2002). Estudio del estadio de cambio y estado emocional en jugadores patológicos. Adicciones, 14, 145-150.

Mark, M.E. y Lesieur, H.R. (1992). A feminist critique of problem gambling research. British Journal of Addiction, 87, 549-565.

McCormick, R.A., Russo, A.M., Ramirez, L.F. y Taber, J.I. (1984). Affective disorders among pathological gamblers seeking treatment. American Journal of Psychiatry, 141, 215-218.

McCormick, R.A. y Ramirez, L.F. (1988). Pathological gambling. En J.G. Howells (Ed.), Modern perspectives in psychosocial pathology (pp. 135-157). New York: Brunner/Mazel Inc.

Miguel-Tobal, J.J. (1995). Evaluación de la ansiedad y de los trastornos de ansiedad. En A. Roa (Comp.), Evaluación en Psicología Clínica y de la Salud. Madrid: Ciencias de la Educación Preescolar y Especial.

Ramirez, L.F., McCormick, R.A., Russo, A.M. y Taber, J.I. (1983). Patterns of substance abuse in pathological gamblers undergoing treatment. Addictive Behaviors, 8, 425-428.

Rodríguez-Martos, A. (1986): El diagnóstico del alcoholimo a través de cuestionarios. Barcelona: Fons Informatiu de l'Ajuntament de Barcelona, $n^{\circ} 2$.

Rodríguez-Martos, A. (1989). Estudio piloto estimativo de la prevalencia de juego patológico entre los pacientes alcohólicos que acuden al programa DROSS. Revista Española de Drogodependencias, 14, 265-275.

Shaffer, H.J. y Hall, M.N. (1996). Estimating the prevalence of adolescent gambling disorders: A quantitative synthesis and guide toward standard gambling nomenclature. Journal of Gambling Studies, 12, 193214

Shaffer, H.J., Bilt, J. y Hall, M.N. (1999). Gambling, drinking, smoking and other health risk activities among casino employees. American Journal of Industrial Medicine, 36, 365-378.

Smart, R.B. y Ferris, J. (1996). Alcohol, drugs and gambling in the Ontario adult population. Canadian Journal of Psychiatry, 41, 36-45.

Spielberger, C.D., Gorsuch, R.I. y Lushene, R. (1970). The State-Trait Anxiety Inventory (self-evaluation Questionnaire). Palo Alto, C.A.: Psychologists Press (versión española, TEA, 1986). 
Spunt, B., Lesieur, H., Hunt, D. y Cahill, L. (1995). Gambling among methadone patients. International Journal of Addictions, 30, 929-962.

Spunt, B., Dupont, I., Lesieur, H.R., Liberty, H.J. y Hunt, D. (1998). Pathological gambling and substance misuse: A review of the literature. Substance Use \& Misuse, 33, 2535-2560.

Steinberg, M.A., Kosten, T.A. y Rounsaville, B.J. (1992a). Cocaine abuse and pathological gambling. The American Journal on Addictions, 1, 121-132.

Steinberg, M.A., Kosten, T.A. y Rounsaville, B.J. (1992b). Pathological gamblers in methadone treatment: A comparison between men and womwn. Journal of Gambling Studies, 12, 431-449.

Stinchfield, R., Cassuto, N., Winters, K. y Latimer, W. (1997). Prevalence of gambling among Minnesota Public School students in 1992 and 1995. Journal of Gambling Studies, 13, 25-48.
Vázquez, C. y Sanz, J. (1999). Fiabilidad y validez de la versión española del Inventario para la Depresión de Beck de 1978 en pacientes con trastornos psicológicos. Clínica y Salud, 10, 59-81.

Villa, A., Becoña, E. y Vázquez, F.L. (1997). Juego patológico con máquinas tragaperras en una muestra de escolares de Gijón. Adicciones, 9, 195-208.

Volberg, R.A. (1994). The prevalence and demographics of pathological gamblers: Implications for Public Health. American Journal of Public Health, 84, 237-241.

Volberg, R.A. y Steadman, H.J. (1988). Refining prevalence estimates of pathological gambling. American Journal of Psychiatry, 145, 502-505.

Zimmerman, M.D. (1994). Interview guide for evaluating DSM-IV psychiatric disorders and the mental status examination. Philadelphia: Psychiatric Press Products. 
